\title{
Sintomas Vocais e Efeitos da Hidratação Após Treinamento Artístico em Centros de Tradições Gaúchas
}

\author{
Kerolin Duarte Gomes, ${ }^{1}$ Chenia Caldeira Martinez, ${ }^{2}$ Márcio Pezzini França ${ }^{1}$
}

\begin{abstract}
RESUMO
Este estudo visou a identificar sintomas vocais e analisar os efeitos da hidratação com água na voz de participantes de invernadas artísticas dos Centros de Tradições Gaúchas (CTGs). Trata-se de um ensaio clínico não randomizado, unicego, dois braços de intervenção, realizado com 88 indivíduos participantes de CTGs de Porto Alegre-RS, com idades entre 17 e 48 anos, de ambos os sexos. Quatro CTGs foram selecionados e convidados para a pesquisa, cada um deles sendo randomizado para um grupo de intervenção: Grupo Hidratação (GH) ou Grupo Controle (GC). O GH incluiu 53 indivíduos que receberam orientações sobre saúde vocal e tiveram a oferta monitorada de água durante o período de ensaio da invernada. O GC contou com 35 indivíduos que seguiram com a rotina normal de ensaio, recebendo apenas orientações sobre saúde em geral (não relacionadas à voz). As avaliações foram feitas nos períodos pré e pós-ensaio imediatos, por meio da Escala de Sintomas Vocais e do Questionário de Sinais e Sintomas Vocais. Observou-se que 42 indivíduos (47,7\% da amostra) apresentaram elevado número de sintomas vocais, indicando possível risco para distúrbio vocal. As mulheres apresentaram maiores escores, e os sujeitos que participavam da invernada há mais tempo relataram menos sintomas. Não houve diferença significativa entre os grupos, exceto para a variável idade $(p=0,005)$. Após o ensaio, observou-se diminuição do sintoma "dificuldade para cantar ou falar baixo" no GH e aumento deste no GC ( $p=0,005)$. Os dados indicam que a amostra estudada pode estar em riscos para distúrbios vocais e a hidratação mostrou efeitos benéficos nestes indivíduos.
\end{abstract}

Palavras-chave: voz; canto; dança; Fonoaudiologia; Saúde; cultura.

VOICE SYMPTOMS AND EFFECTS OF HYDRATION AFTER ARTISTIC TRAINING IN “CENTRO DE TRADIÇÕES GAÚCHAS”

\section{ABSTRACT}

This study aimed to identify voice symptoms and to analyze the effects of water hydration on the voice of participants of artistic groups of a Centro de Tradições Gaúchas (CTGs). It was a clinical trial, non-randomized, single-blinded, two-arm of intervention with 88 participants of CTGs from City of Porto Alegre-Rio Grande do Sul, aged 17 to 48 years old, of both sexes. Four CTGs were selected and invited to the research, and randomized to intervention groups: Hydration Group (GH) and Control Group (CG). The GH included 53 individuals who received vocal health guidelines and had a monitored supply of water during the artistic training period. The CG included 35 individuals who followed the normal training routine, receiving only guidance on general health aspects (not about voice). Evaluations were made in the immediate pre and post-trial periods by Voice Symptom Scale and Voice Symptoms/Sign Questionnaire. It was observed that 42 individuals ( $47.7 \%$ of the sample) had a high number of vocal symptoms before the test, indicating risk for voice disorders. Women had higher scores, and the subjects who participated in the CTG group longer had fewer symptoms. There was no statistically significant difference in the comparison between groups, except for the age variable $(p=0.005)$. After the training, there was a decrease in the symptom "difficulty in singing or speaking low" in $\mathrm{GH}$ and an increase in the CG $(p=0.005)$. The results indicate that the studied sample may be at risk for vocal disorders and hydration showed beneficial effects of these individuals.

Keywords: voice; singing; dancing; speech-language pathology and audiology. health. culture.

RECEBIDO EM: 22/10/2019

MODIFICAÇÕES SOLICITADAS EM: 29/12/2020

ACEITO EM: 29/4/2021

\footnotetext{
${ }^{1}$ Universidade Federal do Rio Grande do Sul - UFRGS.

${ }^{2}$ Autora correspondente. Universidade Federal do Rio Grande do Sul, Instituto de Psicologia - UFRGS. Rua Ramiro Barcelos. Rio Branco. Porto Alegre/RS, Brasil. CEP 90035-003. http://lattes.cnpq.br/0142067535581447. https://orcid.org/00000002-5421-7366. chenia.martinez@gmail.com
} 


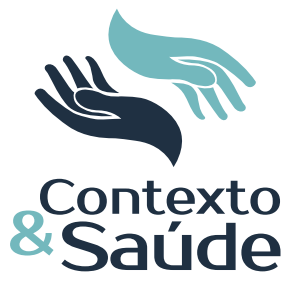

\section{INTRODUÇÃO}

Os Centros de Tradições Gaúchas (CTGs) são entidades que compõem um organismo social de natureza cívica, cultural, literária, artística e folclórica no Rio Grande do Sul (RGS). Para tanto, precisam estar de acordo com o Movimento Tradicionalista Gaúcho, uma associação civil, sem fins lucrativos, com circunscrição em todo o território nacional (MTG, 2018). Entre as diversas atividades realizadas pelos CTGs, destacam-se os grupos de danças tradicionalistas, nomeadas de invernadas artísticas (RODRIGUES, 2016).

As invernadas artísticas são grupos de dança não remunerados, porém consideradas semiprofissionais, que participam de diversas competições artísticas. Entre estas competições destaca-se o Encontro de Arte e Tradição (Enart), considerado um dos maiores festivais da América Latina, envolvendo premiações diversas que determinam quem são os "melhores artistas" do tradicionalismo gaúcho a cada ano (VALLEJOS, 2013; BIANCALANA, 2014).

A prática das danças tradicionalistas está se tornando cada vez mais exigente e para melhorar suas performances nas competições, os grupos ensaiam cerca de duas a três vezes por semana, com duração média de duas horas por dia, completando aproximadamente seis horas de ensaio, podendo intensificar a frequência das atividades em períodos próximos às competições principais, como o Enart. Durante os ensaios e apresentações, os dançarinos fazem uso da voz cantada, mesmo sendo considerados grupos essencialmente de dança e não de canto. Ressalta-se que não há a interrupção das danças, conforme classificadas em seus ciclos coreográficos, para a execução do canto, isto ocorre de forma simultânea (CÔRTES; LESSA, 1997).

Diante do exposto, entende-se ser de fundamental importância a investigação de possíveis alterações ou sintomas vocais nestes indivíduos, bem como a promoção de ações de saúde vocal, uma vez que fazem uso da voz cantada associada a atividades corporais de grande intensidade. Para manter a saúde vocal, principalmente para quem canta, programas de higiene são pensados de modo que diminuam o abuso e uso indevido da voz, além de incentivar a constante hidratação, haja vista que a água é um componente vital para o desempenho das funções corporais e vocais, que auxilia na diminuição das queixas vocais e também melhora a produção vocal dos indivíduos que utilizam a voz de modo intenso (VERDOLINI-MARKSON; SANDAGE; TITZE, 1994; SIQUIERA et al., 2016; WYK et al., 2016).

Destaca-se, ainda, a falta de estudos na literatura que tenham investigado aspectos de saúde vocal nesta população, que pode estar em risco de desenvolver sintomas e alterações vocais. Assim sendo, esta pesquisa teve o objetivo de investigar a presença de sintomas vocais em integrantes de invernadas artísticas de CTGs, bem como comparar a autopercepção destes sintomas antes e depois de uma intervenção fonoaudiológica envolvendo hidratação indireta com ingestão de água associada a um ensaio de dança.

Editora Unijuí - Revista Contexto \& Saúde - ISSN 2176-7114 - v. 21, n. 43, jul./set. 2021 


\section{MÉTODOS}

\section{Tipo e Local de estudo}

Trata-se de um ensaio clínico com grupo controle e com randomização em grupos, realizado com amostra de conveniência. Os participantes eram integrantes de invernadas artísticas de Centros de Tradições Gaúchas de Porto Alegre. Foram selecionados quatro CTGs por conveniência, que foram posteriormente randomizados em dois grupos de intervenção, Grupo Hidratação e Grupo Controle, por programa de computador (random.org).

\section{Participantes}

Quanto aos sujeitos da amostra, foram incluídos 88 indivíduos com idades entre 17 e 48 anos, de ambos os sexos, participantes ativos das invernadas artísticas. Foram excluídos aqueles que autorreferiram diagnóstico médico de alteração laríngea associado à queixa de disfonia no momento da aplicação dos questionários iniciais ou que se recusaram a participar da intervenção.

\section{Procedimentos de Avaliação e Intervenção}

As avaliações incluíram ficha clínica e os protocolos Escala de Sintomas Vocais (ESV) (MORETI et al., 2011) e Questionário de Sinais e Sintomas Vocais (QSSV) (ROY et al., 2004), que foram aplicados em ambos os grupos pré-ensaio. Ao final do ensaio apenas o QSSV, junto a um recordatório de ingesta de água, durante o período de atividade, foram aplicados. Além disso, cabe ressaltar que ambos os grupos receberam, no momento pré-intervenção, orientações de saúde fonoaudiológica com duração de 15 minutos. O GH recebeu orientação sobre saúde vocal, benefícios do aquecimento da voz e da hidratação principalmente em situações de uso profissional da voz. O GC recebeu orientações gerais que envolviam postura, audição e equilíbrio, ou seja, informações não relacionadas à saúde vocal.

A ESV é uma escala validada e composta por 30 itens que exploram três domínios: limitação, emocional e físico, sendo direcionada para identificação de sintomas vocais e alteração de voz. Cada item é respondido em escala likert, com possibilidades que variam entre "nunca", "raramente", "às vezes", "quase sempre" e "sempre". Quanto maior o escore, maior a percepção dos sintomas vocais, constituindo um indicativo de alteração vocal quando o valor for maior ou igual a 16 pontos. A QSSV contém uma lista de 14 sinais e sintomas vocais, autorreferida pelo indivíduo, sendo considerado um ponto para cada sintoma presente e se esse sintoma possui relação ou não com a atividade laboral. A ficha clínica e o recordatório de água foram elaborados pelos pesquisadores, a fim de abordar todos os aspectos relevantes para a presente pesquisa.

O Grupo Hidratação recebeu oferta sistemática de água, em temperatura ambiente, com quantidade de ingestão total de $600 \mathrm{ml}$ por participante, sempre supervisionada pelo pesquisador. $O$ consumo de água ocorreu nos seguintes momentos do ensaio: 1) antes do início $(200 \mathrm{ml}) ; 2$ ) após 30 minutos de ensaio $(100 \mathrm{ml})$; 3) após 60 minutos de ensaio (100ml); 4) no momento de encerramen- 
to do ensaio (200ml). Já o Grupo Controle viveu a rotina natural do ensaio, sem nenhum tipo de intervenção ou oferta monitorada de água por parte dos pesquisadores, no entanto alguns participantes ingeriram água de forma espontânea, o que foi posteriormente mensurado com o questionário, aplicado ao final do ensaio, que tinha o objetivo recordatório.

\section{Análise dos Dados e Aspectos Éticos}

Os dados iniciais foram analisados por meio de estatística descritiva, apresentados em frequência absoluta e relativa. A normalidade foi testada por meio do teste Kruskal Wallis, e as variáveis não paramétricas foram apresentadas em mediana e intervalo interquartil. Os dados seguintes foram analisados por meio dos testes Mann-Whitney, teste t para amostras independentes e Exato de Fisher. $\mathrm{O}$ teste de Equações de Estimativas Generalizadas foi realizado para medir o efeito da interação da intervenção experimental (hidratação) com o tempo e também entre os grupos Hidratação e Controle. O nível de significância adotado foi de $5 \%(p \leq 0,05)$ pelo software SPSS versão 18.0.

O presente estudo buscou uma análise de dados baseada também na "análise de intenção de tratar", visando a possibilitar uma aproximação da intervenção aplicada ao contexto da prática clínica real, preservando a distribuição aleatória dos sujeitos e suas características clínicas. O projeto de pesquisa foi aprovado pelo Comitê de Ética em Pesquisa em Seres Humanos (CAAE 97156818.5.0000.5334). Os participantes foram informados quanto aos objetivos da pesquisa e assinaram o Termo de Consentimento Livre e Esclarecido (TCLE).

\section{RESULTADOS}

Os dados de caracterização da amostra estão presentes na Tabela 1. Foi realizada a comparação das principais variáveis clínicas para verificar a homogeneidade da amostra, entre elas "idade", "sexo" e "uso profissional da voz" entre os Grupos Hidratação e Controle, observando-se diferença estatisticamente significativa na variável idade, indicando que o Grupo Controle incluiu, aleatoriamente, sujeitos mais velhos e que não houve diferença entre os grupos nas demais variáveis.

Tabela 1 - Dados de caracterização da amostra

\begin{tabular}{lcccc}
\hline & $\begin{array}{c}\text { Total } \\
(\mathrm{n}=88)\end{array}$ & $\begin{array}{c}\text { Grupo } \\
\text { Hidratação } \\
(\mathrm{n}=53)\end{array}$ & $\begin{array}{c}\text { Grupo } \\
\text { Controle } \\
(\mathrm{n}=35)\end{array}$ & $\begin{array}{c}\text { Valor } \\
\text { de } \mathrm{p}\end{array}$ \\
\hline $\begin{array}{l}\text { Idade - média } \pm \mathrm{DP} \\
\text { Sexo }-\mathrm{n}(\%)\end{array}$ & $25,9 \pm 6,5$ & $24,2 \pm 7,3$ & $28,4 \pm 5,3$ & $0,005^{*}$ \\
Feminino & $44(50,0)$ & $25(47,2)$ & $19(54,3)$ & $0,663^{* *}$ \\
Masculino & $44(50,0)$ & $28(52,8)$ & $16(45,7)$ & \\
$\begin{array}{l}\text { Uso profissional voz - } \\
\text { mediana (p25-p75) }\end{array}$ & $6,0(0,2-8,0)$ & $6,0(1,0-8,0)$ & $5,0(0,0-8,0)$ & $0,085^{* *}$
\end{tabular}

Editora Unijuí - Revista Contexto \& Saúde - ISSN 2176-7114 - v. 21, n. 43, jul./set. 2021 


\begin{tabular}{|c|c|c|c|}
\hline $\begin{array}{l}\text { Tempo de dança - mediana } \\
\text { (p25-p75) }\end{array}$ & $5,0(2,0-12,0)$ & $4(11,0-2,0)$ & $10(2,0-18,0)$ \\
\hline Horas de ensaio - média $\pm D P$ & $8,0 \pm 2,3$ & $8,0 \pm 2,4$ & $8,0 \pm 2,0$ \\
\hline Horas de sono - média $\pm D P$ & $6,8 \pm 1,5$ & $7,1 \pm 1,7$ & $6,4 \pm 1,2$ \\
\hline Tabagismo - n (\%) & $6(6,8)$ & $3(5,6)$ & $3(8,5)$ \\
\hline Ingestão de álcool.- n (\%) & $61(69,3)$ & $36(67,9)$ & $25(71,4)$ \\
\hline $\begin{array}{l}\text { Alimentação gordurosa - n } \\
\text { (\%) }\end{array}$ & $33(37,5)$ & $21(39,6)$ & $12(34,2)$ \\
\hline
\end{tabular}

Legenda: Dados apresentados em frequência absoluta e relativa, média e desvio padrão para variáveis paramétricas, mediana e quartis para variáveis não paramétricas. *teste t para amostras independentes; **Exato de Fisher. Valores significativos ( $p \leq 0,05)$.

\section{Fonte: Elaborada pelos autores.}

Os sintomas vocais pré-intervenção foram analisados isoladamente e em comparação com variáveis clínicas dos sujeitos, considerando a amostra total. A mediana de escore no QSSV foi de 2 sintomas, e de 14 sintomas na ESV. Ainda, destaca-se que 42 indivíduos (47,7\% da amostra) apresentaram número de sintomas vocais acima do ponto de corte na ESV. Observou-se relação estatisticamente significativa entre as variáveis "sexo feminino" e "escore aumentado de sintomas vocais", indicando que mulheres apresentam maior número de sintomas, por meio do teste Mann-Whitney.

Quanto à comparação dos sintomas vocais com variáveis clínicas, houve significância estatística com relação ao número de sintomas vocais e ingestão de bebida alcoólica, com que esta variável sendo atribuída aos indivíduos que autorreferiram ingerir bebida alcoólica apenas um dia da semana. Ainda neste contexto, do total da amostra, apenas 7 indivíduos $(7,9 \%)$ referiram a ingestão por três dias ou mais durante a semana e 28 sujeitos ( $31,8 \%$ da amostra) referiram não consumir bebida alcoólica semanalmente.

Tabela 2 - Comparação das variáveis clínicas e sintomas vocais pré-ensaio

\begin{tabular}{ccc}
\hline & ESV & QSSV \\
& Mediana (p25-p75) & Mediana (p25-p75) \\
\hline Escore total & 14,00 & 2,0 \\
Sexo & & \\
F & $20(10-26,8)$ & $2(1-4)$ \\
M & $11(5-22,8)$ & $1(0-3)$ \\
P & $0,015^{*}$ & 0,109 \\
Profissional Voz & & \\
Sim & $20(9-28)$ & $2(0-4)$ \\
Não & $12(7,5-22)$ & $2(0-3)$ \\
P & 0,139 & 0,355 \\
Simgestão de água pré-intervenção & $14(9-26)$ & $2(0-4)$ \\
Não & $18(1,5-21)$ & $2(0-2)$ \\
P & 0,244 & 0,368 \\
Tabagismo & $16,5(3-24)$ & $2,5(0-4,5)$ \\
Sim & $14(8-25,2)$ & $2(0-3,2)$ \\
Não & &
\end{tabular}

Editora Unijuí - Revista Contexto \& Saúde - ISSN 2176-7114 - v. 21, n. 43, jul./set. 2021 


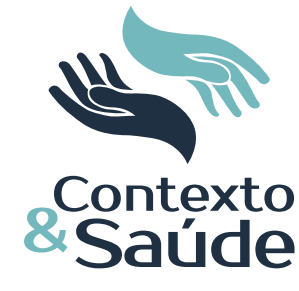

Voz cansada depois do uso por um curto tempo

Problemas para cantar ou falar baixo

Dificuldade para projetar a voz

Dificuldade para cantar agudo

Desconforto ao falar

Voz monótona

Esforço para falar

Garganta seca

Dor na garganta

Pigarro

Gosto ácido ou amargo na boca

Dificuldade para engolir

Instabilidade ou tremor na fala
0,661

0,735

$$
\begin{gathered}
13(7-22) \\
24(10-30) \\
0,047^{*}
\end{gathered}
$$

$2(0-3)$

$2(1-5)$

0,167

$\begin{array}{cc}13(9-24,5) & 2(0,5-3) \\ 16(7-27) & 2(0-4) \\ 0,918 & 0,944\end{array}$

Legenda: *Valores significativos ( $\mathrm{p} \leq 0,05)$; Teste Mann-Whitney; ESV - Escala de Sintomas Vocais; QSSV ocais.

Fonte: Elaborada pelos autores.

Na Tabela 3 apresenta-se a comparação dos sintomas vocais do QSSV em

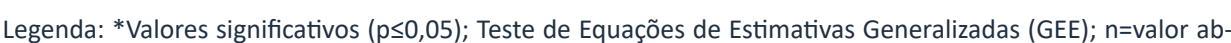
soluto; \%=valor em porcentagem.

Fonte: Elaborada pelos autores. 


\section{DISCUSSÃO}

Este estudo foi desenvolvido com uma população que potencialmente apresenta riscos de desenvolver sintomas e alterações vocais. Para tanto, investigou-se a presença desses sintomas vocais e o efeito da hidratação antes e depois dos ensaios, por meio de comparação da autopercepção dos indivíduos entre os grupos.

Diversos estudos consideram a hidratação um hábito importante a ser seguido, visto que é de suma importância para a prevenção dos distúrbios vocais, pois reduz a viscosidade das pregas vocais e o esforço fonatório, promovendo, consequentemente, um menor atrito durante a vibração. Este mecanismo contribui para a diminuição das queixas vocais e favorece uma melhor produção vocal (UEDA; DOS SANTOS; OLIVEIRA, 2008; VERDOLINI-MARKSON; SANDAGE; TITZE, 1994; SIQUEIRA et al., 2016; MORETI; ZAMBON; BEHLAU, 2016). Os achados no presente estudo corroboram com a literatura, posto que o resultado com maior significância estatística é o da hidratação controlada, realizada no grupo experimental, que mostrou a redução do sintoma vocal na variável "dificuldade para cantar ou falar baixo", ocorrendo de modo inverso no Grupo Controle, conforme Tabela 3.

Na literatura, observa-se que não há definição sobre a quantidade ideal de água que seja necessária para manter a hidratação das pregas vocais (VERDOLINI-MARKSON; SANDAGE; TITZE, 1994; WYK et al., 2016). Alguns estudos, no entanto, recomendam a ingestão de água de forma fracionada, no decorrer do dia, para uma boa hidratação e que antes de utilizar a voz por longos períodos, o indivíduo beba pelo menos um copo de água e continue se mantendo hidratado enquanto faz uso da voz, ainda que sem uma dosagem específica (VERDOLINI-MARKSON; SANDAGE; TITZE, 1994; BEHLAU; PONTES; MORETI, 2017). No Grupo Controle, grande parte dos participantes referiu ingerir água antes $(54,2 \%)$ e durante $(85,7 \%)$ o período de ensaio, mesmo sem orientações e controle da quantidade que ingeriram. Este fato pode explicar a pouca diferença dos sintomas analisados entre os grupos na Tabela 3.

Os achados analisados na comparação entre os grupos Hidratação e Controle mostraram que não houve diferença entre eles nas variáveis sexo e uso profissional da voz, evidenciando que os grupos tiveram distribuição semelhante. A variável idade, contudo, apresentou diferença com significância estatística entre os grupos estudados, porém destaca-se que para este estudo a diferença de idade (24,2 e 28,4 anos) não apresenta significância clínica, na medida em que os indivíduos encontram-se na mesma faixa etária, classificados como adultos jovens.

Com relação ao consumo de bebidas alcoólicas, os achados corroboram com estudos realizados com outras populações que utilizam a voz como meio de expressão, quando os indivíduos relataram ingerir bebidas alcoólicas com frequência, porém sem exageros (GIANNINI; LATORRE; FERREIRA, 2012; AQUINO; TELES, 2013; PUHL et al., 2017). O consumo de bebidas alcoólicas é comum no país, visto que dados divulgados pelo Instituto Brasileiro de Geografia e Estatística (IBGE, 2016) mostraram que cerca de $30 \%$ da população geral consome bebida alcoólica ao menos uma vez na semana.

Editora Unijuí - Revista Contexto \& Saúde - ISSN 2176-7114 - v. 21, n. 43, jul./set. 2021 


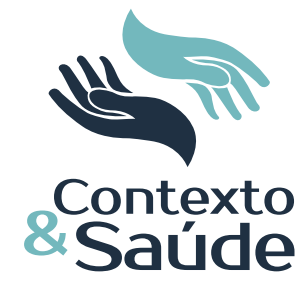

Outro ponto importante a ser destacado é a inclusão de sujeitos que faziam uso de tabaco em ambos os grupos estudados. Sabe-se que o tabagismo pode provocar prejuízos na qualidade vocal dos sujeitos, podendo interferir nos resultados. Visto, no entanto, que este estudo é baseado na análise por intenção de tratar (FLETCHER; FLETCHER; FLETCHER, 2014), optou-se pela não exclusão destes sujeitos na análise. Além disso, vale ressaltar que a distribuição foi igual entre os sujeitos tabagistas nos dois grupos, sendo que do total de seis indivíduos, três foram alocados no GH e três no GC. Ainda, na Tabela 2, são comparados os resultados dos indivíduos tabagistas com escores da ESV e QSSV, não havendo diferença estatisticamente significativa entre eles na quantidade de sintomas vocais. Estes resultados reforçam que a variável tabagismo, provavelmente, não interferiu nos resultados identificados por este estudo.

A respeito dos sintomas vocais, observa-se que apesar de a mediana de sintomas na ESV ter sido abaixo do ponto de corte, podemos ainda considerar que os valores apresentados estão elevados em comparação com os dados de pessoas sem disfonia (AMARAL et al., 2017). No presente estudo, 44 indivíduos $(47,7 \%$ da amostra total) apresentaram escores maiores que 16 pontos nesta escala, representando um número elevado de indivíduos com sintomas vocais. A quantidade de sintomas vocais nesta população pode ser atribuída a diversos fatores, tais como pouca informação, preparo e orientações quanto ao uso da voz e saúde vocal (PENTEADO; SILVA; MONTEBELLO, 2015).

A literatura recomenda que cantores amadores, a exemplo dos dançarinos de invernadas, necessitam de noções sobre saúde vocal, assim como os demais profissionais da voz, a fim de evitar a instalação de alterações vocais futuras (ZlMMER; CIELO; FERREIRA, 2012). Foi observado, todavia, que as variáveis "queixa de voz rouca" no QSSV e "usar a voz profissionalmente" não apresentam associação estatisticamente significativa, indicando que a queixa relativa à qualidade vocal rouca não esteve associada ao uso ocupacional da voz.

No presente estudo as mulheres apresentaram escore maior de sintomas vocais que os homens. Este dado corrobora a literatura, que mostra o sexo feminino mais predisposto a alterações vocais pela relação com as características hormonais, anatômicas e de menor proporção glótica das laringes femininas (SATALOFF, 1981; RIBEIRO et al., 2012; DASSIE-LEITE; LOURENÇO; BEHLAU, 2011). Importante ressaltar que, apesar de esta amostra ter sido homogênea na variável sexo, é comum o número elevado de mulheres em comparação a homens em grupos de danças dos CTGs. Assim sendo, este grupo de mulheres pode apresentar maior risco para sintomas e/ou alterações vocais quando em situações de uso da voz e dança simultâneas.

Destaca-se ainda que determinadas danças tradicionais requerem o uso da voz cantada simultaneamente à dança, de acordo com seus ciclos coreográficos (CÔRTES; LESSA, 1997; RODRIGUES, 2016). Alguns autores destacam que o ato de cantar se compara a uma atividade esportiva que requer bom condicionamento e interação coordenada de diversas funções físicas, demandando do indivíduo grande energia física e maior fôlego para realizar a atividade (SATALOFF, 1981; ZIMMER; CIELO; FERREIRA, 2012). Corroborando os achados na literatura, observa-se que os indivíduos que dançam há mais tempo em invernadas artísti-

Editora Unijuí - Revista Contexto \& Saúde - ISSN 2176-7114 - v. 21, n. 43, jul./set. 2021 
cas apresentam maior condicionamento, visto que apresentam menor escore e quantidade de sintomas vocais relatados quando comparados aos indivíduos que dançam há pouco tempo.

Por fim, observou-se mudança na quantidade de sintomas vocais pré e pós-ensaio em ambos os grupos, porém sem diferença significativa. Este achado sugere que ambas as abordagens provocaram, de alguma forma, mudança na autopercepção vocal dos participantes, independentemente de qual tenha sido o tempo de intervenção. O que corrobora a literatura, uma vez que, apesar de apenas uma abordagem indireta, com um dia de intervenção não possibilitar diferenciação significativa, o conhecimento sobre hábitos vocais, saudáveis ou prejudiciais, auxilia na identificação de fatores que podem estar relacionados a alterações vocais (RIBEIRO et al., 2012; ANHAIA et al., 2014; SANTOS; BORREGO; BEHLAU, 2015; BEHLAU; PONTES; MORETI, 2017).

\section{CONCLUSÕES}

Observou-se nesta pesquisa que a hidratação constante durante ensaios diminui a sensação de dificuldade para cantar e falar baixo na população estudada, que pode estar em risco para desenvolver alteração vocal devido à quantidade de pessoas que apresentaram índices elevados de sintomas vocais, principalmente mulheres. Outros fatores que corroboram os achados neste estudo podem ser o pouco conhecimento sobre o uso adequado da voz e outros cuidados quanto à saúde vocal.

Sugerem-se estudos que acompanhem por um período longo, de vários ensaios, com amostras maiores e pareadas nesta população. Além disso, propõe-se outras intervenções e investigações que considerem diferentes aspectos de avaliação, como a qualidade vocal de cada dançarino.

\section{REFERÊNCIAS}

AMARAL, Amanda Corrêa do et al. Desconforto do trato vocal em professores após atividade letiva. Codas, v. 29, n. 2, e20160045, 2017.

ANHAIA, Tanise Cristaldo et al. Efeitos de duas intervenções em professores com queixas vocais. Audiol., Commun. Res., v. 19, n. 2, p. 186-193, 2014.

AQUINO, Fernanda Salvatico de; TELES, Lídia Cristina da Silva. Autopercepção vocal de coristas profissionais. Rev. Cefac, v. 15, n. 4, p. 986-993, 2013.

BEHLAU, Mara; PONTES, Paulo; MORETI, Felipe. Higiene vocal - cuidando da voz. 5. ed. Rio de Janeiro: Editora Revinter, 2017.

BIANCALANA, Gisela Reis. Danças tradicionalistas riograndenses, gênero e memória. Conceição/Concept, v. 3, n. 2, p. 23-33, 2014.

CÔRTES, João Carlos Paixão; LESSA, Luís Carlos Barbosa. Manual de danças tradicionais gaúchas. 8. ed. Brasil: Irmãos Vitale, 1997.

DASSIE-LEITE, Ana Paula; LOURENÇO, Luciana; BEHLAU, Mara. Relação entre dados ocupacionais, sintomas e avaliação vocal de operadores de telesserviços. Rev. Soc. Bras. Fonoaudiol., v. 16, n. 1, p. 59-63, 2011.

FLETCHER, Robert H.; FLETCHER Suzanne W.; FLETCHER, Grant S. Epidemiologia clínica: elementos essenciais. 5. ed. Porto Alegre: Artmed, 2014.

Editora Unijuí - Revista Contexto \& Saúde - ISSN 2176-7114 - v. 21, n. 43, jul./set. 2021 
GIANNINI, Susana Pimentel Pinto; LATORRE, Maria do Rosário Dias de Oliveira; FERREIRA, Leslie Piccolotto. Distúrbio de voz e estresse no trabalho docente: um estudo caso-controle. Cad. Saúde Pública, v. 28, n. 11, p. 2.115-2.124, 2012.

IBGE. Instituto Brasileiro de Geografia e Estatística. Pesquisa nacional de saúde: 2013 indicadores de saúde e mercado de trabalho: Brasil e grandes regiões. Rio de Janeiro: Coordenação de Trabalho e Rendimento. 2016. Disponível em: http://www.saude.gov.br/ noticias/911-indicadores-de-saude/41421-pesquisa-nacional-de-saude-pns. Acesso em: 21 out. 2019.

MORETI, Felipe et al. Equivalência cultural da versão Brasileira da Voice Symptom Scale: VoiSS. J. Soc. Bras. Fonoaudiol., v. 23, n. 4, p. 398-400, 2011.

MORETI, Felipe; ZAMBON, Fabiana; BEHLAU, Mara. Conhecimento em cuidados vocais por indivíduos disfônicos e saudáveis de diferentes gerações. Codas, São Paulo, v. 28, n. 4, p. 463-469, 2016.

MTG. Movimento Tradicionalista Gaúcho. Histórico, o que é MTG? Disponível em: http:// www.mtg.org.br. Acesso em: 10 nov. 2018.

PENTEADO, Regina Zanella; SILVA, Noelle Bernardi da; MONTEBELLO, Maria Imaculada de Lima. Voz, estresse, trabalho e qualidade de vida de técnicos e preparadores físicos de futebol. Codas, v. 27, n. 6, p. 588-597, 2015.

PUHL, Andrea Estér et al. Tabagismo e ingesta alcoólica: prevalência em professores, cantores, teleoperadores e atores. Distúrb. Comum., v. 29, n. 4, p. 683-691, 2017.

RIBEIRO, Vanessa Veis et al. Identificação de problemas vocais enfrentados por cantores de igreja. Rev. Cefac, v. 14, n. 1, p. 90-96, 2012.

RODRIGUES, Karen Domingues. Danças tradicionais gaúchas: (de)compondo sua movimentação. 2016. Trabalho de Conclusão de Curso (Licenciatura em Música) - Universidade Federal de Pelotas, Pelotas, 2016.

ROY, Nelson et al. Voice disorders in teachers and the general population: effects on work performance, attendance, and future career choices. J. Speech Lang Hear Res., v. 47, n. 3, p. 542-551, 2004.

SANTOS, Andréia Cristina Muznlinger dos; BORREGO, Maria Cristina de Menezes; BEHLAU, Mara. Efeito de treinamento vocal direto e indireto em estudantes de Fonoaudiologia. Codas, v. 27, n. 4, p. 384-391, 2015.

SATALOFF, Robert Thayer. Professional singers: The science and art of clinical care. Am. J. Otolaryngol., v. 2, n. 3, p. 251-266, 1981.

SIQUEIRA, Márcia do Amaral et al. Hidratação vocal em profissionais e futuros profissionais da voz. Rev. Cefac, v. 18, n. 4, p. 908-914, 2016.

UEDA, Kelly Hitomi; DOS SANTOS, Leila Zambuze; OLIVEIRA, lara Bittante de. 25 anos de cuidados com a voz profissional: avaliando ações. Rev. Cefac, v. 10, n. 4, p. 557-565, 2008. VALLEJOS, Maitê. Encontro de Arte e Tradição (Enart): o cultivo da tradição cultural gaúcha por meio do festival. 2013. Trabalho de Conclusão de Curso (Curso em Mídia, Informação e Cultura) - Universidade de São Paulo, Escola de Comunicações e Artes, São Paulo, 2013.

VERDOLINI-MARKSON, Katherine; SANDAGE Mary; TITZE Ingo R. Effect of hydration treatments on laryngeal nodules and polyps and related voice measures. J. Voice, v. 8, n. 1, p. 30-47, 1994.

WYK, Liezl Van et al. The Effect of Hydration on the Voice Quality of Future Professional Vocal Performers. J. Voice, v. 31, n. 1, p. 111, e29-111.e36, 2016.

ZIMMER, Valquíria; CIELO, Carla Aparecida; FERREIRA, Fernanda Mariotto. Comportamento vocal de cantores populares. Rev. Cefac, v. 14, n. 2, p. 298-307, 2012. 